\title{
Hypophosphatasia now draws more attention of both clinicians and researchers: A Commentary on prevelance of c. 1559delT in ALPL, a common mutation resulting in the perinatal (lethal) form of hypophosphatasias in Japanese and effects of the mutation on heterozygous carriers
}

\author{
Keiichi Ozono and Toshimi Michigami
}

Journal of Human Genetics (2011) 56, 174-176; doi:10.1038/jhg.2011.6; published online 10 February 2011

$\mathrm{H}$ ypophosphatsia is a skeletal disease due to an inborn error of metabolism characterized by deficient activity of the tissuenonspecific alkaline phosphatase. ${ }^{1,2}$ Alkaline phosphatase (ALP) is a membrane-bound metalloenzyme that consists of a group of isoenzymes encoded by four different gene loci: tissue-nonspecific, intestinal, placental and germ-cell ALP. Tissue-nonspecific alkaline phosphatase is expressed in almost all cells and three organs, liver, bone and kidney, have high activity of ALP. Thus, tissuenonspecific alkaline phosphatase is also called ALP liver/bone/kidney (ALPL). The mouse counter part is called Akp2. Tissuenonspecific alkaline phosphatase hydrolyzes inorganic pyrophosphate, an inhibitor of mineralization, and increases the local concentration of inorganic phosphate. Therefore, hypomineralization of skeleton and rachitic change of bone is associated with hypophosphatasia.

Hypophosphatasia is highly variable in its clinical expression. On the basis of the age of manifestation and its severity, hypophosphatasia is divided into six subtypes (Table 1).

Professor K Ozono is at the Department of Pediatrics, Osaka University Graduate School of Medicine, 2-2 Yamadaoka, Suita, Osaka 565-0871, Japan and T Michigami is at the Department of Bone and Mineral Research Osaka Medical Center and Research Institute for Maternal and Child Health, 840 Murodo-cho, Izumi, Osaka 594-1101, Japan.

E-mail: keioz@ped.med.osaka-u.ac.jp
The most severe form of hypophosphatasia is a perinatal form, which is also called a lethal form. This form presents clinically either before or in the newborn period. The patients with this form exhibit severe defect of bone mineralization including craniotabes, bone deformity, short stature and narrow chest, and suffer from respiratory failure (Figure 1). However, some patients of this form can survive because of advances in neonatology. ${ }^{3}$ Recently, non-lethal benign form of perinatal hypophosphatasia has been recognized, which is associated with no apparent defects of mineralization. ${ }^{4,5}$ An infantile form is characterized by infantile onset and often associated with poor weight gain, hypercalcemia and respiratory difficulties. This form shows still rather high mortality. A childhood form is manifested at the age of 2 or 3 with premature loss of teeth, rachtic change of long bones and mild short stature. An adult form is sometimes associated with pathologic bone fracture and an odonto types means a form of only teeth manifestation. Diagnosis of hypophosphatasia is made on the basis of the clinical features, skeletal X-ray findings and low activity of ALP associated with elevation of its substrate, such as phosphoethanolamine, inorganic pyrophosphate and pyridoxal-5'-phosphate. We described diagnostic criteria of hypophosphatasia proposed by the Japanese Hypophosphatasia Study Group at http://www.bone.med.osakau.ac.jp/english/b5/.
Hypophosphatasia is usually inherited in an autosomal recessive manner, but can be expressed in an autosomal dominant manner in milder forms. ${ }^{6}$ The ALPL gene is located on the short arm of chromosome 1 (1p36.1p34), and contains 12 exons. The product consists of 507 amino acids including residues of the signal peptide. Hypophosphatasia is caused by various mutations in the $A L P L$ gene. Currently, more than 200 mutations of the ALPL gene in patients with hypophosphatasia have been registered in the $A L P L$ gene mutations database (http://www.sesep. uvsq.fr/Database.html). Most mutations described to date are missense mutations $(80 \%)$, and the remainder was several deletion and insertion mutations of one to four nucleotides. Large deletions of the gene are rare $(1.3 \%)$. Correlations of genotype and phenotype have been reported on the basis of clinical data of the patients with hypophosphatasia, enzymatic activity and computerassisted modeling. ${ }^{2}$

We previously reported that the most and the second-most frequent mutations in Japanese patients are T1559del and F310L, respectively. ${ }^{5}$ These mutations are renamed to c.1559delT and p.F327 L, respectively, based on the recent standardized nomenclature. Interestingly, both mutations are unique to Japanese. The common mutations, c.1559delT and p.F327 L, are associated with lethal and the perinatal non-lethal forms of hypophosphatasia, respectively. This is an example of 
Table 1 Subtypes of hypophosphatasia and features

\begin{tabular}{|c|c|c|}
\hline Subtypes & Onset & Features \\
\hline Perinatal lethal & Fetus, neonate & Respiratory failure, severe mineralization defect, convulsion \\
\hline Perinatal non-lethal & Fetus, neonate & Bone deformity \\
\hline Infantile & Infant & Failure to thrive, hypercalcemia \\
\hline Childhood & Child, especially toddlers & Premature loss of teeth, rickets-like changes \\
\hline Adult & Adulthood & Fragile bone \\
\hline Odonto & Not indicated & Teeth involvement only \\
\hline
\end{tabular}

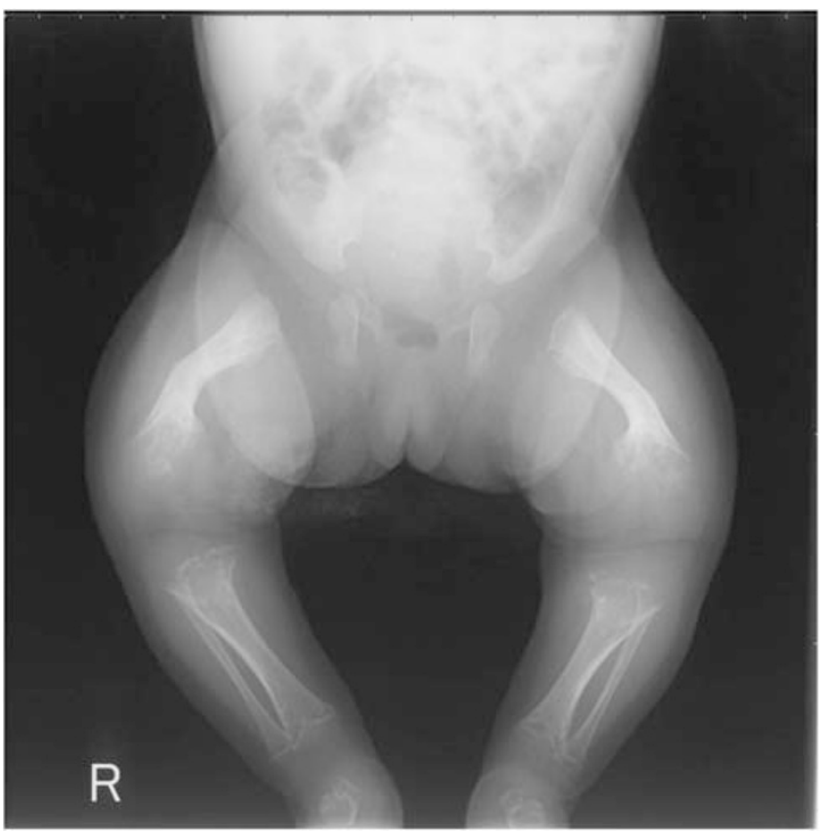

Figure 1 X-ray of legs of a neonate of hypophosphatasia. Severe rachitic changes of metaphysis of long bones are characteristic to hypophosphatasia. Bone deformity is also recognized.

the genotype-phenotype relationship, consistent with the enzymatic activity of the mutant ALP proteins; c.1559delT caused a complete loss of activity, whereas p.F327 L retains some residual activities. Therefore, genotyping patients with hypophosphatasia may help to predict their prognosis. In Europe, the E174K (renamed to p.E191K) mutation is reported to be frequent with a frequency of $31 \%$ in patients with mild hypophosphatasia. ${ }^{7}$ As the E174K is associated with the same rare haplotype, the E174K mutation is surmised to be an ancestral mutation. However, the allele frequency of the E174K mutation is not investigated in normal population. In this issue of the journal, Watanabe et al. ${ }^{8}$ reported prevelance of c. 1559delT in ALPL, a common mutation resulting in the perinatal (lethal) form of hypophosphatasias in Japanese. According to the article, the frequency is one per $480(1 / 480)$, resulting in $1 / 920000$ homozygotes, because de novo mutation seems extremely rare. The frequency indicates more than one patient with this homozygous mutation is born per year in Japan, which has $\sim 1100000$ newborns per year. In our experience of examination of mutation in the $A L P L$ gene in 42 patients with hypophosphatasia, homozygous mutation of c.1559delT was found in $17 \%$ patients. Thus, around seven patients with hypophosphatasia per year may be born in Japan based on the small number of examination. The carrier frequency for the mutant allele is estimated to be $1 / 25$ in the Manitoba Mennonite community in Canada, which has the highest incidence rate for severe form of hypophosphatasia. $^{9}$

There is no established medical treatment to cure hypophosphatasia, but there are some specific treatments for complications of hypophosphatasia. ${ }^{1}$ In severe form of hypophosphasia, patients often suffer from intractable convulsions. This complication was also reported in akp2 $2^{-1-}$ mice. ${ }^{10}$ The convulsion can be controlled by administration of vita- min B6 because abnormal metabolism of vitamin B6 leading to the deficient $\gamma$-aminobutyric acid in brain is observed in akp2 $2^{-1-}$ mice. In an infantile form of hypophosphatasia, patients tend to have hypercalcemia because of low bone formation. Low calcium-containing milk is recommended for hypercalcemia. Recently, treatment with parathyroid hormone 1-34, teriparatide, has been reported to improve bone formation, although its effect is still controversial. ${ }^{11,12}$ Bone marrow transplantation has been reported to treat several patients with hypophosphatasia. ${ }^{13}$ However, a method must be developed that improves the survival of donor mesenchymal cells in patients. Likewise, other congenital enzyme defect disorders, such as Hurler or Hunter disease, recombinant enzyme replacement therapy is being attempted in hypophosphatasia. Recombinant bone-targeted ALP therapy is effective in terms of mineralization and life-span in akp2 $2^{-1-}$ mice. ${ }^{14}$ The therapy is now on clinical trial and expected to be available in near future.

1 Whyte, M. P. Physiological role of alkaline phosphatase explored in hypophosphatasia. Prolonged survival and phenotypic correction of Akp2(-/-) hypophosphatasia mice by lentiviral gene therapy. Ann. N. Y. Acad. Sci. 1192, 190-200 (2010).

2 Mornet, E. Hypophosphatasia. Orphanet. J. Rare. Dis. 2, 40 (2007).

3 Nakamura-Utsunomiya, A., Okada, S., Hara, K ., Miyagawa, S., Takeda, K., Fukuhara, R. et al. Clinical characteristics of perinatal lethal hypophosphatasia: a report of 6 cases. Clin. Pediatr. Endocrinol. 19, 7-13 (2010).

4 Stevenson, D. A., Carey, J. C., Coburn, S. P., Ericson, K. L., Byrne, J. L., Mumm, S. et al. Autosomal recessive hypophosphatasia manifesting in utero with long bone deformity but showing spontaneous postnatal improvement. J. Clin. Endocrinol. Metab. 93, 3443-3448 (2008).

5 Michigami, T., Uchihashi, T., Suzuki, A., Tachikawa, K. Nakajima, S. \& Ozono, K. Common mutations F310L and T1559del in the tissue-nonspecific alkaline phosphatase gene are related to distinct phenotypes in Japanese patients with hypophosphatasia. Eur. J. Pediatr. 164, 277-282 (2005).

6 Müller, H. L., Yamazaki, M., Michigami, T., Kageyama, T., Schönau, E., Schneider, P. et al. Asp361Val mutant of alkaline phosphatase found in patients with dominantly inherited hypophosphatasia inhibits the activity of the wild-type enzyme. J. Clin. Endocrinol. Metab. 85, 743-747 (2000)

7 Hérasse, M., Spentchian, M., Taillandier, A. \& Etienne, $M$. Evidence of a founder effect for the tissue-nonspecific alkaline phosphatase (TNSALP) gene E174K mutation in hypophosphatasia patients. Eur. J. Hum. Genet. 10, 666-668 (2002).

8 Watanabe, A., Karasugi, T., Sawai, H., Naing, B. T., Ikegawa, S., Orimo, H. et al. Prevalence of c.1559delT in $A L P L$, a common mutation resulting in the perinatal (lethal) form of hypophosphatasia in Japanese and effects of the mutation on heterozygous carriers. J. Hum. Genet. 56, 166-168 (2011).

9 Greenberg, C. R., Taylor, C. L., Haworth, J. C., Seargeant, L. E., Philipps, S., Triggs-Raine, B. et al. A homoallelic Gly317->Asp mutation in ALPL causes the perinatal (lethal) form of hypophosphatasia in 
Canadian mennonites. Genomics. 17, 215-217 (1993).

10 Fedde, K. N., Blair, L., Silverstein, J., Coburn, S. P., Ryan, L. M., Weinstein, R. S. et al. Alkaline phosphatase knock-out mice recapitulate the metabolic and skeletal defects of infantile hypophosphatasia. J. Bone. Miner. Res. 14, 2015-2026 (1999).
11 Whyte, M. P., Mumm, S. \& Deal, C. Adult hypophosphatasia treated with teriparatide. J. Clin. Endocrinol. Metab. 92, 1203-1208 (2007).

12 Gagnon, C., Sims, N. A., Mumm, S., McAuley, S. A., Jung, C., Poulton, I. J. et al. Lack of sustained response to teriparatide in a patient with adult hypophosphatasia. J. Clin. Endocrinol. Metab. 95, 1007-1012 (2010).
13 Whyte, M. P., Kurtzberg, J., McAlister, W. H., Steele, A., Coburn, S. P., McAlister, W. H. et al: Marrow cell transplantation for infantile hypophosphatasia. J. Bone. Miner. Res. 18, 624-636 (2003).

14 Millán, J. L., Narisawa, S., Lemire, I., Loisel, T. P., Boileau, G., Leonard, P. et al. Enzyme replacement therapy for murine hypophosphatasia. J. Bone. Miner. Res. 23, 777-787 (2008). 